\title{
Timing is everything: PTTH mediated DHR4 nucleocytoplasmic trafficking sets the tempo of Drosophila steroid production
}

\author{
Kim F. Rewitz ${ }^{1}$ and Michael B. O'Connor ${ }^{2 *}$ \\ 1 Department of Science, Systems and Models, Roskilde University, Roskilde, Denmark \\ ${ }^{2}$ Department of Genetics, Cell Biology and Development, University of Minnesota, Minneapolis, MN, USA
}

Edited by:

Alexander William Shingleton,

Michigan State University, USA

\section{Reviewed by:}

Kirst King-Jones, University of Alberta, Canada

Christen Kerry Mirth, Fundação

Calouste Gulbenkian, Portugal

Wendy Smith, Northeastern

University, USA

${ }^{*}$ Correspondence:

Michael B. O'Connor, Department of

Genetics Cell Biology and

Development, University of

Minnesota, 6-160 Jackson Hall,

Minneapolis, MN 55455, USA.

e-mail:moconnor@umn.edu
During development, multicellular organisms must become sexually mature in order to reproduce. The developmental timing of this transition is controlled by pulses of steroid hormones, but how these pulses are generated have remained unclear? A recent paper shows that in Drosophila larvae, nucleocytoplasmic trafficking of DHR4, a nuclear receptor, in response to prothoracicotropic hormone signaling, is critical for producing the correct temporal pulses of steroid hormones that coordinate the juvenile-adult transition.

Keywords: 20-hydroxyecdysone, DHR4, critical weight, Cyp6t3, metamorphosis
In many animals reaching a species-specific size induces a developmental switch from juvenile growth to reproductive maturity. This transition occurs when a developmental timing program is aligned with permissive checkpoint signals, such as nutritional status and photoperiod, to activate a neuroendocrine circuit that promotes steroid-induced maturation in organisms ranging from flies to humans (Dungan et al., 2006; Tennessen and Thummel, 2011).

In some insects, it is thought that attainment of a proper level of nutrient stores, known as the critical weight, plays a major role in activation of neuroendocrine signal(s) that trigger metamorphosis, a process that produces a reproductively mature adult (McBrayer et al., 2007; Mirth and Riddiford, 2007). Although the mechanisms for assessing size/weight are poorly understood (see however Callier and Nijhout, 2011), the output of achieving critical weight in Drosophila correlates well with production of an early low-level pulse of the steroid hormone ecdysone (E), 8-10 h after ecdysis from the second to the third instar stage. This is followed by at least two other low-level E pulses midway through the third instar stage, and finally by a much larger E peak just as pupariation commences (Warren et al., 2006; Mirth and Riddiford, 2007). The mechanisms responsible for generating the initial critical weightassociated pulse, and the importance of this and the other low-level E pulses for orchestrating the proper timing of the late major larval E pulse, that initiate metamorphosis, have remained quite vague. In a recent paper published in PLoS Biology, Ou et al. (2011) begin to provide some insight into this issue by demonstrating that the trafficking of the nuclear receptor DHR4 between the nucleus and the cytoplasm in response to a brain-derived neuropeptide known as prothoracicotropic hormone (PTTH) controls the dynamics of the E peaks and the timing of metamorphosis.

\section{PTTH SIGNALING AND METAMORPHOSIS}

Previous studies in both Lepidoptera and Drosophila have demonstrated a key role for PTTH in stimulating E production and metamorphosis (Rybczynski, 2005; McBrayer et al., 2007). In Drosophila, ablating the PTTH producing neurons causes substantial developmental delay in the onset of pupariation. In contrast, over-expression of PTTH leads to the opposite phenotype, i.e., early onset or precocious pupariation (McBrayer et al., 2007; Rewitz et al., 2009b). PTTH stimulates E production by binding to its receptor Torso, a receptor tyrosine kinase, which is expressed in prothoracic gland (PG) cells, the endocrine tissue of the larva responsible for E production. Torso activates the Ras/Raf/Mek pathway resulting in the phosphorylation and targeting of phosphorylated Erk (pErk) to the nucleus. Interestingly, expression of an activated form of Ras in the PG induces precocious pupariation similar to what is seen when PTTH is expressed ubiquitously (Rewitz et al., 2009b).

\section{DHR4 IS A KEY MEDIATOR OF PTTH SIGNALING AND IS} ESSENTIAL TO PREVENT PREMATURE METAMORPHOSIS

A major block to a more comprehensive understanding of how the PTTH signal stimulates E production in the PG and times the onset of metamorphosis has been the lack of knowledge concerning downstream mediators of pathway activity, especially potential transcription factors that are targets for phosphorylation by Erk. Previous work on the nuclear receptor DHR4 had shown 
that larvae carrying mutations in this gene undergo precocious maturation, reducing the juvenile growth period and producing small animals (King-Jones et al., 2005) similar to that caused by hyper-activating PTTH signaling. Importantly, Ou et al. (2011) demonstrated that the developmental timing defects exhibited by DHR4 mutants are phenocopied by specific loss of DHR4 in the PG cells and not by knockdown of DHR4 in the fat body, another tissue in which DHR4 shows strong expression. These observations led to the question of whether there might be a functional connection between PTTH signaling and DHR4 activity. As illustrated in Figure 1, they discovered a very interesting inverse correlation between PTTH mediated Erk nuclear localization and the subcellular distribution of DHR4 in the PG cells. In the absence



FIGURE 1 | Prothoracicotropic hormone/DHR4 generate cyclic steroid pulses that drive directional developmental transitions. A model illustrating the mechanisms generating 8-16 h ultradian steroid oscillations. (A) Each cycle is initiated by the release of PTTH from terminals of brain neurons that activates the Ras pathway in the PG. This results in the phosphorylation and nuclear localization of pERK and translocation of DHR4 to the cytoplasm, which derepresses ecdysone biosynthesis. (B) In the presence of PTTH, the DHR4 repressor is displaced from promoters, including Cyp6t3 increasing its expression. During periods without PTTH signaling, nuclear DHR4 represses gene expression, although DHR4 may act as an activator in some cases. pErk, phospho-ERK; PG, prothoracic gland; PRE, PTTH response element; PTTH, prothoracicotropic hormone. Torso: the transmembrane PTTH receptor in the PG. Dashed arrows indicate inactive mechanisms. of PTTH signaling, DHR4 was found primarily in the nucleus, while constitutive PTTH signaling resulted in mainly cytoplasmic DHR4. While it was not shown that Erk directly phosphorylates DHR4, this seems highly likely since simple inspection of the DHR4 primary sequence reveals the presence of several consensus Erk phosphorylation sites.

The inverse correlation of Erk and DHR4 nuclear localization suggests that the primary role of nuclear DHR4 in the PG is to dampen the PTTH signal to prevent early metamorphosis. This hypothesis was confirmed with an epistasis experiment where over-expression of DHR4 in the PG was found to suppress the early pupation phenotype of activated Ras expression in the PG. Furthermore, using carefully timed heat shock pulse-driven DHR4 RNAi, the authors were able to demonstrate that the crucial time at which DHR4 is required to prevent precocious metamorphosis is around the time of the early third instar when the critical weight and the low-level E pulses are determined. Intriguingly, they found that in DHR4-depleted larvae, the E titer rose faster and did not decline as in the controls leading to a step-like appearance in the E titer profile instead of a discrete pulse. Taken together, these data indicate that DHR4 is a key target of PTTH signaling in the PG and provide the first compelling evidence that the small early third instar E peak is essential for setting the tempo of larval development.

\section{Cyp6t3: A KEY ECDYSONE BIOSYNTHETIC COMPONENT AND TARGET OF DHR4 REPRESSION?}

The observation that PTTH signaling appears to upregulate E titers by removing DHR4 from the nucleus leads to the obvious questions of what genes DHR4 regulates and how they impact E synthesis or degradation? To begin to address this question, $\mathrm{Ou}$ et al. (2011) used a microarray approach in which they specifically isolated RNA from carefully timed wildtype Drosophila ring glands, a tissue that contains the PG cells, and compared the profiles to similarly timed glands in which the level of DHR4 expression was reduced by RNAi knockdown. This technically demanding experiment was able to identify a small number of genes $(\sim 50)$ that showed either enhanced or reduced expression upon loss-of-DHR4 function. The most interesting among these, with respect to E metabolism, were several Cyp gene family members that code for P450 enzymes of which several are required for either synthesis or degradation of E (Gilbert and Warren, 2005; Rewitz et al., 2010). Intriguingly, none of the known E biosynthetic P450s showed much change in expression in response to loss-of-DHR4 function, at least not at the time points that were examined. However, two members of the Cyp6 family, including Cyp6t3, showed significant upregulation upon DHR4 depletion. The authors focused their analysis on Cyp6t3 since its expression was restricted to the ring gland and showed oscillations that correspond to the nucleocytoplasmic oscillations of DHR4. Knockdown of Cyp6t3 in the PG was found to produce E deficiency phenotypes that could be rescued by feeding larvae $\mathrm{E}$ or one of several E biosynthetic precursors. These experiments tentatively place Cyp6t3, along with Spook, another P450, and Shroud a short chain dehydrogenase, at the mysterious "black box" step that catalyzes conversion of 7-dehydrocholesterol to diketol by an unknown mechanism. Lastly, epistasis experiments showed that 
upregulation of Cyp6t3 contributes to the developmental timing defect of DHR4 loss since knockdown of Cyp6t3 in a DHR4 mutant background suppressed the precocious pupation phenotype.

Despite these compelling findings suggesting a key role for Cyp6t3 as a new E biosynthetic component, there are some puzzling aspects. First, Cyp $6 t 3$ shows no strong conservation among insects, which is unusual, compared to the hitherto characterized E biosynthetic enzymes. For Cyp6t 3 there are numerous related Cyp family members in different insect species, however, they are not clear orthologs as can be found for all the other key Cyp E biosynthetic enzymes. Further de novo synthesis of $\mathrm{E}$ is required for embryogenesis and oogenesis. While the expression of Cyp6t3 in ovaries and embryos was not reported, data available through the Flybase does not support much expression in these tissues, although it may be very low as is observed in the PG. A full analysis of the null mutant phenotype will be necessary to address a possible role in embryogenesis and either clonal or RNAi knockdown in follicle cells to address a role in ovaries. Even if cyp6t3 is not required for E biosynthesis in either of these tissues, this might once again simply reflect a division of labor with a related Cyp6 family member that is expressed during embryogenesis and in the ovary. In fact, this same situation occurs for Spook and Spookier, two essential ecdysteroidogenic P450s which, interestingly enough, are also implicated at the black box step. In this case, spook is expressed in embryos and ovaries and its function is dispensable in larvae, while spookier is expressed in the larval PG and its function is not required in embryos and presumably the ovary as well. However, in this example Spook and Spookier are clear paralogs; no similarly related Cyp6 relatives exist in Drosophila. A third issue is the very low-level expression of Cyp6t3 in the PG. As the authors suggest, this may indicate that Cyp6t 3 is one of the rate-limiting factors for E production, but over-expression did not accelerate development as might be expected if this was the case. Thus, although these experiments strongly implicate Cyp6t3 in E biosynthesis, additional experiments to clarify its precise biochemical activity are needed to fully understand its role in regulating the E concentration.

\section{WHAT'S NEXT?}

The work described by Ou et al. (2011) undoubtedly demonstrates that DHR4 is an important component of the network regulating the timing of the steroid cues that drive the gene circuit underlying time-directional development in Drosophila. Like all good breakthroughs however, the reported experiments raise as many interesting questions as they answer. For example, what aspect of the altered E titer profile in DHR4 mutants is key to accelerating development? Is it the more rapid kinetics of its production, its higher plateau level, or lack of decline that is important? Also, does DHR4 affect the oscillation of the two subsequent low-level third instar E peaks as well as the large late major larval peak, and if so, do these also act as timing signals or provide some other function

\section{REFERENCES}

Callier, V., and Nijhout, H. F. (2011). Control of body size by oxygen supply reveals size-dependent and size-independent mechanisms of molting and metamorphosis. Proc. Natl. Acad. Sci. U.S.A. 108, 14664-14669.

Dungan, H. M., Clifton, D. K., and Steiner, R. A. (2006). Minireview:

such as behavioral switches? Perhaps this can be addressed using additional and more refined temporal knockdown of either DHR4 or PTTH signaling components. Indeed, the whole issue of how the ultradian cycles in Erk/DHR4 nuclear localization are timed is something that needs to be examined. While previous work has shown that PTTH transcription cycles with an 8 -h periodicity, the Erk/DHR4 nuclear cycles and the third instar E pulses are not in synchrony with an 8-h periodicity, with the exception of the first small E pulse (McBrayer et al., 2007). Clearly, some other process besides $P T T H$ transcription is setting the rhythmicity of PTTH/DHR4 signaling.

How DHR4 nucleocytoplasmic cycling is accomplished is also unclear. As the authors point out, simply shuttling a stable DHR4 out of the nucleus in response to PTTH signaling would not seem to be consistent with the ability to induce rapid phenotypes by RNAi. The alternative is that nuclear DHR4 degradation is enhanced by PTTH signaling, and its reuptake into the nucleus is inhibited until the PTTH signal is terminated. In this regard it would be interesting to determine if removal of DHR4 from the nucleus provides negative feedback to terminate the PTTH signal perhaps by inducing phosphatases that dephosphorylate components of the Raf/Mek/Erk pathway. In the Manduca PG, for example, ERK levels decline after 1-h of continuous exposure to PTTH (Rybczynski et al., 2001; Rewitz et al., 2009a). Simply monitoring the Erk nuclear residence time in DHR4 knockdown larvae might help address this possibility.

One last interesting experiment would be to examine the consequence of double RNAi-mediated knock down of both torso and DHR4 in the PG which would presumably result in the loss of both Erk and DHR4 cycling. Upon initial reflection, it might seem that this manipulation would have to affect timing since, presumably, the large late-larval E peak would not occur in the absence of PTTH. However, the answer might be surprising if, for example, there was a steady increase in $\mathrm{E}$ in the double RNAi larvae, perhaps simply as the result of gland growth, and the larvae were able to integrate the total E signal over time. There is precedence for this ability in Manduca, where a prolonged continuous small E dose can have the same effect as a larger pulsed dose (Nijhout, 1976). Signal integration of a steady E ramp up may enable the larvae to still initiate metamorphosis with close to normal timing. The results by $\mathrm{Ou}$ et al. (2011) provide important insights into regulation of the steroid pulse generator that, from an evolutionary perspective, seems to be a key part of the neuroendocrine system coordinating the juvenile-adult transition in animals.

\section{ACKNOWLEDGMENTS}

We thank Naoki Yamanaka and Mary Jane Shimell for helpful comments on the manuscript. This work was supported in part by a grant from the Danish Council for Independent Research, Natural Science to Kim F. Rewitz and R01-GM093301-02 from NIGMS to Michael B. O’Connor.

kisspeptin neurons as central processors in the regulation of gonadotropin-releasing hormone secretion. Endocrinology 147, 1154-1158.
Gilbert, L. I., and Warren, J. T. (2005). A molecular genetic approach to the biosynthesis of the insect steroid molting hormone. Vitam. Horm. 73, 31-57. 
King-Jones, K., Charles, J. P., Lam, G., and Thummel, C. S. (2005). The ecdysone-induced DHR4 orphan nuclear receptor coordinates growth and maturation in Drosophila. Cell 121, 773-784.

McBrayer, Z., Ono, H., Shimell, M., Parvy, J. P., Beckstead, R. B., Warren, J. T., Thummel, C. S., Dauphin-Villemant, C., Gilbert, L. I., and O'Connor, M. B. (2007). Prothoracicotropic hormone regulates developmental timing and body size in Drosophila. Dev. Cell 13, 857-871.

Mirth, C. K., and Riddiford, L. M. (2007). Size assessment and growth control: how adult size is determined in insects. Bioessays 29, 344-355.

Nijhout, H. F. (1976). The role of ecdysone in pupation of Manduca sexta. J. Insect Physiol. 22, 453-463.

Ou, Q., Magico, A., and King-Jones, K. (2011). Nuclear receptor DHR4 controls the timing of steroid hormone pulses during Drosophila development. PLoS Biol. 9, e1001160. doi:10.1371/journal.pbio. 1001160

Rewitz, K. F., Larsen, M. R., LobnerOlesen, A., Rybczynski, R., O'Connor, M. B., and Gilbert, L. I. (2009a). A phosphoproteomics approach to elucidate neuropeptide signal transduction controlling insect metamorphosis. Insect Biochem. Mol. Biol. 39, 475-483.

Rewitz, K. F., Yamanaka, N., Gilbert, L. I., and O'Connor, M. B. (2009b). The insect neuropeptide PTTH activates receptor tyrosine kinase torso to initiate metamorphosis. Science 326, 1403-1405.

Rewitz, K. F., Yamanaka, N., and O'Connor, M. B. (2010). Steroid hormone inactivation is required during the juvenile-adult transition in Drosophila. Dev. Cell 19, 895-902.

Rybczynski, R. (2005). "The prothoracicotropic hormone," in Comprehensive Molecular Insect Science, eds
L. I. Gilbert, K. Iatrou, and S. Gill (Oxford: Elsevier), 61-123.

Rybczynski, R., Bell, S. C., and Gilbert, L. I. (2001). Activation of an extracellular signal-regulated kinase (ERK) by the insect prothoracicotropic hormone. Mol. Cell. Endocrinol. 184, 1-11.

Tennessen, J. M., and Thummel, C. S. (2011). Coordinating growth and maturation - insights from Drosophila. Curr. Biol. 21, R750 R757.

Warren, J. T., Yerushalmi, Y., Shimell, M. J., O'Connor, M. B., Restifo, L. L., and Gilbert, L. I. (2006). Discrete pulses of molting hormone, 20-hydroxyecdysone, during late larval development of Drosophila melanogaster: correlations with changes in gene activity. Dev. Dyn. 235, 315-326.

Conflict of Interest Statement: The authors declare that the research was conducted in the absence of any commercial or financial relationships that could be construed as a potential conflict of interest.

Received: 01 November 2011; paper pending published: 16 November 2011; accepted: 06 December 2011; published online: 27 December 2011.

Citation: Rewitz KF and O'Connor MB (2011) Timing is everything: $P T T H$ mediated DHR4 nucleocytoplasmic trafficking sets the tempo of Drosophila steroid production. Front. Endocrin. 2:108. doi: 10.3389/fendo.2011.00108

This article was submitted to Frontiers in Experimental Endocrinology, a specialty of Frontiers in Endocrinology.

Copyright (c) 2011 Rewitz and O'Connor. This is an open-access article distributed under the terms of the Creative Commons Attribution Non Commercial License, which permits non-commercial use, distribution, and reproduction in other forums, provided the original authors and source are credited. 